\title{
A second golden age for proteinases
}

The meeting of different cultures, more often than not an enriching experience, can sometimes be fraught with difficulties. And so it seemed at a recent conference on proteinases ${ }^{*}$ where a heterogeneous gathering of cell, molecular and structural biologists - both academic and industrial, sometimes stumbled on the unfamiliar cues provided by the diverse mix of vocational backgrounds and biological disciplines. More importantly, though, the meeting was a timely reminder of the rejuvinated interest in proteinases, both from the point of view of basic science and medicine. Hans Neurath (University of Washington, Seattle) justifiably dubbed this latest boom in research 'the second golden age for proteinases'.

Proteinases are involved in a wide range of biological processes: digestion, defence, precursor processing, generation of antigenic peptides, as well as the business of intracellular protein degradation which is important in the regulation of protein activity. And should one of these processes go awry, the consequences can be devastating for the organism in question.

The range of medical applications for proteinase inhibitors, outlined by Eugene Cordes (Sterling Winthrop) is broad; they have uses in the treatment of infectious diseases caused by bacteria and viruses, inflammation, arthritis, hypertension, emphysema and cancer, for example. Strategies for drug design are to a large extent dictated by the available biochemical or structural information on the receptor and/ or the nature of any existing lead compounds (Irwin Kuntz, University of California at San

* Keystone Symposium: Structural and molecular biology of protease function and inhibition, Santa Fe, New Mexico, 5-12 March 1994.
Francisco). In the absence of such information random screening is the most obvious way forward. Establishment of lead compounds opens up the possibility of similarity searches, analysis of structureactivity relationships, and so on. Where information on the structure of the receptor, preferably complexed with inhibitor, is available the iterative process of drug design can yield highly effective inhibitors.

The meeting reported on a range of drugs being developed as inhibitors of proteinases. Proteolytic processing is crucial during the lifecycles of all organisms and provides a target for antiviral, antimicrobial and antiparasitic drugs. The single-stranded RNA viruses, such as polio and HIV, translate the genome into a single, long poly-protein which is then cleaved into its constituent parts, for example.

The rat poison warfarin seems an unlikely lead for an HIV proteinase inhibitor but Keith Watenpaugh (The Upjohn Company allowed to speak at the last moment by upper management and the patent lawyers) described how their design work has taken them from a molecule with a micromolar inhibition constant to one in the subnanomolar range and which is $90 \%$ bioavailable with a half-life of the order of 16 hours. Others (Joel Huff, Merck Research Laboratories; Dale Kempf, Abbott Laboratories; Noel Roberts, Roche Products Ltd) told of similar design efforts that tackled the problem of specificity, solubility, bioavailability, drug metabolism and safety. Some of these drugs are now in phase II and phase III clinical trials and the results look promising.

Drug evasion by HIV is a notorious problem and again structure-based studies provide an insight into the basis of such resistance (Ben Dunn, University of Florida). 
While some mutations can be readily explained, by stearic hindrance or loss of energetically favourable interactions, the effect of others, often distance from the drugbinding site and with no obvious effect on structure, are a complete mystery. Thus while these studies are starting to lay down more general guidelines of desirable properties in drugs, it is clear that we are still a long way from understanding all the factors that drive ligand binding and determine bio-availability and clearance rates in the patient for example; drug design is still a perilous business.

Not all proteinase inhibitors are manmade, of course. Hirudin, a small protein found in the medicinal leech is a potent anticoagulant, as a result of its inhibition of thrombin. The molecule has been used as the basis for designing a bidentate inhibitor: a carboxy-terminal fragment of hirudin that binds to a site distant from the active site on thrombin has been tethered by a flexible linker to an active-site-binding tetrapeptide (John Maraganore, Biogen, Inc). The new inhibitor, with the moniker Hirulog ${ }^{\mathrm{TM}}$, is considerably more effective than heparin in the treatment of patients undergoing angioplasty.

The activity of other proteinases is being nurtured and encouraged, rather than inhibited. Tissue plasminogen activator (tPA) is an important therapeutic agent in the control of thrombosis, but the treatment has its drawbacks, among them a number of side effects and the requirement of perfusion of the drug over several hours. William Bennet (Genentech) described the generation, using an extensive - and essentially random mutagenesis screen, of a modified tPA with a higher activity, especially against more refractory clots, a lower clearance time, and a reduction in the severity of various side effects. The new tPA molecule, administered as a bolus, has already proved its efficacy in clinical trials.

Reversing the peptide bond cleavage reaction of proteinases would provide a peptide-ligation activity. Such a modified enzyme has been engineered and used to build a fully active RNase from a series of short peptides (James Wells, Genentech). The peptide ligase can also be used to incorporate unnatural amino acids into proteins.

Denis Selkoe (Harvard University) reminded us that 4 million people in the USA and 17-20 million people worldwide suffer from Alzheimer's disease. Deposition of the $39-43$ residue amyloid $\beta$ peptide $(A \beta P)$ in neuritic plaques in the brain seem to be central to the disease phenotype. The proteinase activities, christened $\beta$ - and $\gamma$ secretase, that generate $A \beta P$ from the amyloid precursor protein $(\mathrm{A} \beta \mathrm{PP})$ are as yet unidentified, as is the $\alpha$-secretase activity that catalyses an alternative cleavage, liberating the extracellular domain of A $\beta$ PP. In the absence of the proteinase(s) that do the business researchers are characterizing the parameters that define the various cleavage activities in the hope that they will provide clues as to the identity of the proteinase(s) and the treatment of the disease (Selkoe; Todd Golde, Case Western Reserve University; Sungram Sisodia, Johns Hopkins University). Indeed it would seem that inhibitors that block the $\beta$ secretase activity and reduce levels of $A \beta P$ are to hand (Sinhda Sinha, Athena Neurosciences Inc.) although the nature or derivation of these inhibitors was not revealed, again reminding the audience of the academic/industrial divide.

Another moment of tension was provided by the request of one of the organisers (Charlie Craik, University of California at San Francisco) that people refrain from photographing slides of inhibitors shown by the speakers. But to dwell overlong on these incidents would give a misleading impression of the meeting. The exchange of ideas among scientists, industrial and academic - cell biologist and structural biologist, was both exciting and encouraging.

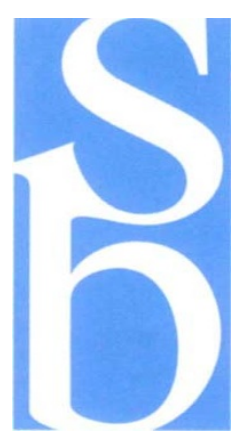

